\title{
Influenza Sentinel Surveillance System in Surb Astvatsamayr Medical Center, 2013-15
}

\author{
Liana Torosyan*, Lilit Avetisyan and Artavazd Vanyan \\ Department of Epidemiology of Special Dangerous and Airborne diseases, National Center of Diseases Control and Prevention, \\ Ministry Of Health, Yerevan, Armenia
}

\section{Objective}

The goal of this study was to identify gaps in the severe acute respiratory infection sentinel surveillance system at Surb Astvatsamayr Medical Center.

\section{Introduction}

Influenza is a priority in Armenia. There are two influenza surveillance systems in Armenia: population and sentinel. The medical center (MC) has been included in sentinel surveillance since 2012. In 2015 a study was undertaken to identify gaps in severe acute respiratory infection (SARI) sentinel surveillance system in Surb Astvatsamayr MC.

\section{Methods}

Medical records and reporting forms of SARI cases were generated for individuals meeting the case definition and analyzed for age groups, risk factors, sentinel surveillance detection methods, laboratory conformation, number of days hospitalized and reporting.

\section{Results}

In 2014, 3016 patients were admitted in the hospital with ARI, of whom 2982 were younger than 18 years. During the 2014-2015 influenza season (week 40, 2014-week 20, 2015), 77 swabs have been taken in total, of which five were influenza positive (4 B and 1 AH1N1). Also in the 2013-2014 influenza season, five samples tested positive (all influenza A). Sixty-one (48\%) patients with respiratory disease met the WHO SARI case definition (2011), $84(66 \%)$ of all reviewed patients would have met the SARI case definition. The numbers for the ICU (25 records reviewed) do not reflect the actual percentage of patients admitted with respiratory symptoms. The 33 additional cases taken from the sampling logbook were mainly hospitalized in the ICU. Influenza tests were performed on 34 patients (mainly ICU), five were positive for influenza (four B--all adults - and one AH1N1), and four tested positives for other respiratory pathogens (two RSV, one RV, one BV). All influenza positives had fever or a history of fever and $80 \%$ met the WHO SARI case definition (2011). Non-sampled cases generally have fewer reported symptoms, but still $44 \%$ of cases fits the WHO SARI case definition (2011).

\section{Conclusions}

The percentages of patients meeting the WHO SARI 2011 case definition and the WHO SARI 2014 definition shows that they were mainly caused by the absence of shortness of breath in the SARI 2014 definition 52\% (2011) vs 66\% (2014) in Surb Asvatsamayr. A large number of children from Neonatal and Children's departments fulfil the SARI case definition and could potentially be swabbed in addition to ICU patients. There are gaps in WHO SARI case definitions. The sentinel surveillance system should be improved.
Comparison of departments and number of patients meeting the WHO SARI case definition

\begin{tabular}{|c|c|c|c|c|c|}
\hline \multirow{2}{*}{ Department } & \multicolumn{2}{|c|}{ Met 2011 WHO SARI } & \multicolumn{2}{|c|}{ Met 2014 WHO SARI** } & \multirow{2}{*}{ Total } \\
\cline { 2 - 5 } & Yes $(\%)$ & No $(\%)$ & Yes $(\%)$ & No $(\%)$ & \\
\hline ICU & $23(72)$ & $9(28)$ & $25(78)$ & $7(22)$ & 32 \\
\hline Neonatal & $18(42)$ & $25(58)$ & $21(49)$ & $22(51)$ & 43 \\
\hline Children's wards & $21(46)$ & $25(54)$ & $32(70)$ & $14(30)$ & 46 \\
\hline Adult therapy & $4(67)$ & $2(33)$ & $6(100)$ & $0(0)$ & 6 \\
\hline Total & $61(48)$ & $66(52)$ & $84(66)$ & $43(34)$ & 127 \\
\hline \multicolumn{2}{|r}{} \\
\hline
\end{tabular}

Keywords

SARI; case definition; sentinel surveillance; Armenia

\section{*Liana Torosyan}

E-mail: liana_torosyan@mail.ru 